\title{
Minimally invasive versus conventional exposure for total hip arthroplasty: a systematic review and meta-analysis of clinical and radiological outcomes
}

\author{
Toby O. Smith $\cdot$ Vicky Blake $\cdot$ Caroline B. Hing
}

Received: 6 May 2010/Revised: 2 June 2010 /Accepted: 3 June 2010 /Published online: 18 June 2010

(C) Springer-Verlag 2010

\begin{abstract}
Over the past decade, minimally invasive surgery has gained popularity as a means of optimising early postoperative rehabilitation and increasing patient satisfaction and cosmesis following total hip arthroplasty (THA). However, this surgical exposure has also been associated with increased risk of iatrogenic nerve injury and implant mal-positioning due to limited visibility compared to conventionally larger surgical incisions. The purpose of this meta-analysis was to compare the outcomes of these two surgical exposures. A systematic review of the published and unpublished literature was conducted to include all randomised and non-randomised controlled trials comparing the clinical and radiological outcomes of minimally invasive and conventional THA procedures. In total, 28 studies met the eligibility criteria and included 2,849 hips, i.e. 1,428 minimally invasive compared to 1,421 conventional THAs. The meta-analysis of the current evidence base showed that minimally invasive THA is associated with a significantly increased risk of transient lateral femoral cutaneous nerve palsy $(p=0.006)$ with no significantly better outcome.
\end{abstract}

\footnotetext{
T. O. Smith $(\square)$

Faculty of Health, Queen's Building, University of East Anglia, Norwich NR4 7TJ, UK

e-mail: toby.smith@uea.ac.uk

V. Blake

Physiotherapy Department,

Norfolk and Norwich University Hospital,

Colney Lane,

Norwich NR4 7UY, UK

C. B. Hing

Department of Trauma and Orthopaedic, St George's Hospital, Tooting, London SW17 0QT, UK
}

\section{Introduction}

Total hip arthroplasty (THA) is the treatment of choice for degenerative changes of the hip joint. The traditional and still most commonly used approaches for primary THA are the posterior approach and direct lateral approach [1-3]. Whilst the recovery and early postoperative outcomes of this procedure have improved over the last 20 years, there remains great interest in accelerated rehabilitation and improving functional outcomes whilst reducing the surgical scar following a THA.

The minimally invasive surgical (MIS) exposure in THA surgery was developed to reduce postoperative bleeding, speed patient recovery and improve the early clinical results [1]. Minimally invasive THA has been defined as an incision length of 10-12 $\mathrm{cm}$ or less either with a single or double incision approach [4-8]. Surgeons have suggested that the smaller skin incision, with reduced soft tissue trauma to muscles, tendons and other soft tissues surrounding the hip should result in less postoperative pain, enhance the patient experience and reduce the length of hospital stay [9-12].

Detractors of MIS have suggested that the approach reduces the operative visualisation thus predisposing patients to implant mal-positioning with an increased risk of dislocation, implant loosening and early failure, in addition to an increased risk of neurovascular complications and excessive skin trauma $[13,14]$.

A previous meta-analysis suggested that there was little difference in the clinical or radiological outcomes following MIS compared to standard exposure THA [15]. However, this study only included randomised and quasi-randomised controlled trials. The purpose of this systematic review was to appraise the entire evidence base to compare the clinical and radiological outcomes of 
patients who have undergone a traditional exposure to a MIS exposure for THA. The primary aim of the systematic review was to determine whether MIS is superior to a conventional exposure with reference to short- and longterm outcomes.

\section{Materials and methods}

Search strategy

All PRISMA compliant searches were performed by TS and $\mathrm{CH}$. The primary search was of published literature using the electronic databases AMED (1985 to April 2010), British Nursing Index (1985 to April 2010), CINHAL (1982 to April 2010), EMBASE (1974 to April 2010) and MEDLINE (1950 to April 2010) using the Ovid search platform. In addition, Scopus, Biomed Central, Zetoc and the Cochrane Library databases were searched. The broad MeSH terms and Boolean operators ("minimally invasive") AND ("hip") AND ("replacement" OR "arthroplasty") were adopted for each database search.

Secondary searches of the unpublished (grey) literature were conducted by searching the electronic databases Open SIGLE (System for Information on Grey Literature in Europe), the WHO International Clinical Trials Registry Platform, Current Controlled Trials, UKCRN Portfolio Database, National Technical Information Service and the UK National Research Register Archive from their inception to April 1, 2010. Conference proceedings were also searched from the British Orthopaedic Association Annual Congress, European Federation of National Associations of Orthopaedics and Traumatology (EFORT) and the British Hip Society to April 2010.

The reference lists from all full text papers included in the review were scrutinised to identify any initially omitted studies. Finally, the corresponding author from each included study was contacted to identify any further studies not previously identified.

\section{Eligibility criteria}

All randomised controlled trials (RCTs) and non-randomised controlled trials (nRCT) comparing the clinical and/or radiological outcomes of THA using a standard exposure to a MIS exposure were included. All trials comparing the exposure method, irrespective of whether computer navigation systems were employed in the MIS surgical arm were included. All trials were included irrespective of their publication status, language, sample size, subject age, indication for surgery, duration of follow-up or surgical approach (i.e. lateral or posterior) undertaken. We excluded all cadaver or animal studies, and those studies assessing exposure method with hip resurfacing or hemi-arthroplasty. We also excluded all trials which used multiple incisions for their MIS rather than a single surgical exposure.

Study selection

The title and abstract for each identified citation were independently screened by two reviewers (TS, $\mathrm{CH}$ ) in relation to the eligibility criteria. Full texts were ordered for those studies which appeared to satisfy these criteria and reviewed independently to determine final inclusion.

\section{Data extraction}

Data from the full text reports were extracted by one reviewer (TS) using a standardised data extraction form, and verified by a second reviewer (VB). The data extracted included: sample size, study design, subject age, gender, THA prosthesis, number of surgeons operating, surgical technique, incision approach, clinical, radiological and complication rate results and follow-up period. The corresponding authors from each included study were contacted to obtain any missing data if required.

\section{Outcome}

The primary outcome for this study was Harris hip score (HHS). Secondary clinical outcomes included: surgical duration, blood loss, pain, requirement for blood transfusion, length of hospital stay, Oxford hip score (OHS) and Western Ontario and McMaster Universities osteoarthritis index score (WOMAC). Radiological secondary outcomes included: cup inclination angle, stem alignment (varus/valgus) angle, leg length discrepancy, femoral offset, incidence of cup positioning (35-55 valgus) and the incidence of stem positioning $\left(0-5^{\circ}\right.$ valgus). Complications included: the incidence of heterotopic ossification, deep and superficial infection, fracture, deep vein thrombosis (DVT), dislocation, haematoma formation, requirement for revision surgery, component loosening, wound complications and the incidence of iatrogenic nerve palsy.

\section{Quality assessment}

Study methodological quality was assessed according to the PEDro critical appraisal tool. This 11-item critical appraisal tool is designed to evaluate comparability between the groups, method of randomisation, blinding and statistical analysis of RCTs. This instrument has previously demonstrated reliability and validity $[16,17]$. 
Data synthesis and analysis

All meta-analyses were performed with the Review Manager software (RevMan Version 5.0; Nordic Cochrane Centre, Copenhagen, Denmark) using the Mantel-Haenszel method [18]. Publication bias was assessed using a funnel plot of the most frequently reported outcome.

Meta-analysis was performed when no substantial heterogeneity in study methodology was observed. Specific statistical heterogeneity was evaluated through $\mathrm{Chi}^{2}$ and $\mathrm{I}^{2}$ statistical tests. When $\mathrm{Chi}^{2}$ was $p<0.05$, and $\mathrm{I}^{2}<20 \%$ indicating low statistical heterogeneity [19], a fixed effect model was used. A random effect model was adopted when $\mathrm{Chi}^{2}$ was $p>0.05$, and $\mathrm{I}^{2}>20 \%$.

Binary data was analysed using risk ratios (RR) with 95\% confidence intervals (CI). Continuous data was assessed with mean differences (MD) or, where different scales or tools are used to measure the same outcome, standardised mean differences with $95 \%$ CI. A probability of $p<0.05$ was considered as statistically significant.

Sub-group analyses were conducted to assess outcomes with and without the assistance of computer navigation for MIS compared to traditional exposure. However, since only one study used computer navigation-assisted surgery for a small proportion of their patients, we did not consider it necessary to undertake a sub-group analysis of this variable.

\section{Results}

\section{Search results}

A total of 534 abstracts and titles were reviewed. Of these 28 satisfied the eligibility criteria and were included in the review (Fig. 1). This included $16 \mathrm{nRCTs}$ and 12 RCTs. Two studies were identified reporting the same cohort. In this instance we included both papers but only analysed the data for each outcome measure once. One study presented the results of two separate surgical approaches using MIS and conventional exposures; both were included in separate analyses [20].

The funnel plot diagram of surgical duration indicated limited evidence of small study exclusion and publication bias with a slightly asymmetrical plot with few studies plotted on the right base of the funnel (Fig. 2).

\section{Quality assessment}

The results of the PEDro review are presented in Table 1. This indicated that there was considerable variability in the evidence base. Whilst the majority of papers defined their cohorts, as previously stated, only 12 RCTs were identified. Of these, only four concealed the randomisation procedure adequately. A power calculation was used to base the sample size in six studies. Furthermore, only five studies presented both outcome measure and demographic characteristics to allow a full assessment of baseline comparability before the trial began. Only three trials attempted to blind subjects to groups allocation. Whilst surgeon blinding would have been inappropriate in this study design, 16 studies did not blind their assessors to patient group. Ten studies were able to report the outcomes of a minimum of $85 \%$ of their starting cohorts; although 13 trials described analysing results through intention-to-treat principles. All studies appropriately used inferential statistics to compare the findings between their experimental cohorts, and all but eight presented both mean and standard deviation or range values to provide an idea of point and variance data from their dataset.

\section{Cohort characteristics}

The demographic characteristics of each study cohort are presented in Table 2. The dataset included 2,825 patients who underwent 2,849 THA procedures. This included 1,428 MIS, with a mean age of 61.8 (standard deviation [SD] 3.9) years including 609 males and 602 females; six studies did not document the gender of their cohort. This group was compared to 1,421 conventional exposure THAs with a mean age of 61.5 (SD 4.9) years, consisting of 610 males and 610 females; six papers did not state the gender of their cohorts.

The most commonly used THA MIS approach was the mini-posterior performed in 12 studies. The most commonly adopted conventional THA approach was the standard posterior approach used in seven studies. Computer navigation surgery was performed in some cases in one study [21]. Follow-up period ranged from five days $[22]$ to five years $[9,23]$.

Primary outcome analysis

There was no significant difference in HHS recorded for the MIS compared to the conventional exposure THA (MD 1.49; 95\% CI $-0.08,3.06 ; p=0.06$; Fig. 3). A difference of less than 2 points would also not be considered a clinically significant difference [24, 25].

Secondary outcome analysis

\section{Clinical outcomes}

As anticipated there was a significantly smaller surgical incision length following MIS compared to conventional THA with a mean difference of $8.0 \mathrm{~cm}(p<0.0001$; Table 3$)$. There was however no statistically significant difference in 
Fig. 1 PRISMA chart

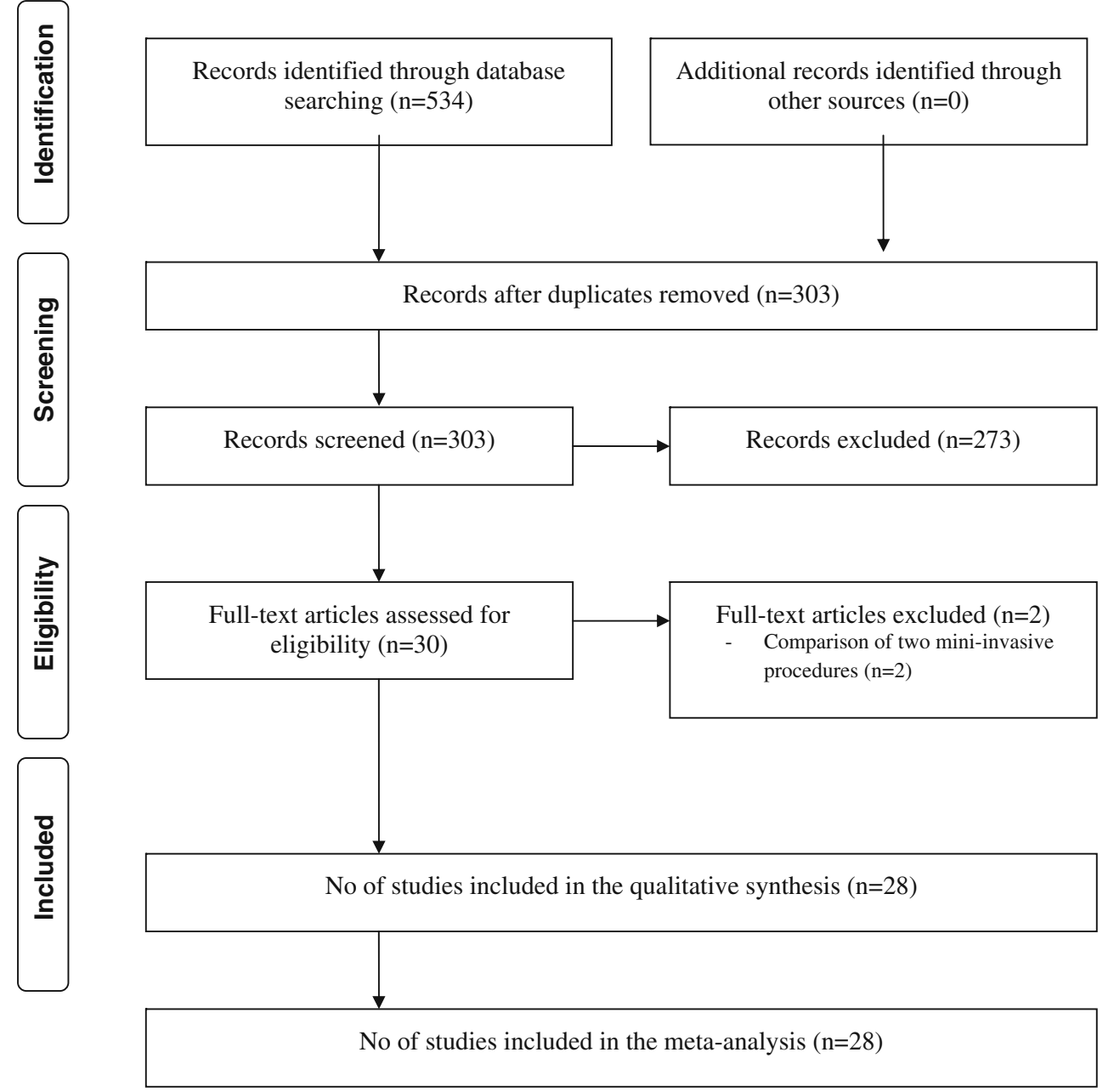

surgical duration between MIS and conventional exposure (MD 4.65 minutes; 95\% CI $-9.45,0.15 ; p=0.06$ ). Whilst there was statistically less perioperative blood loss in the MIS group compared to conventional THA $(p<0.001$; Table 3), there was no statistically significant difference

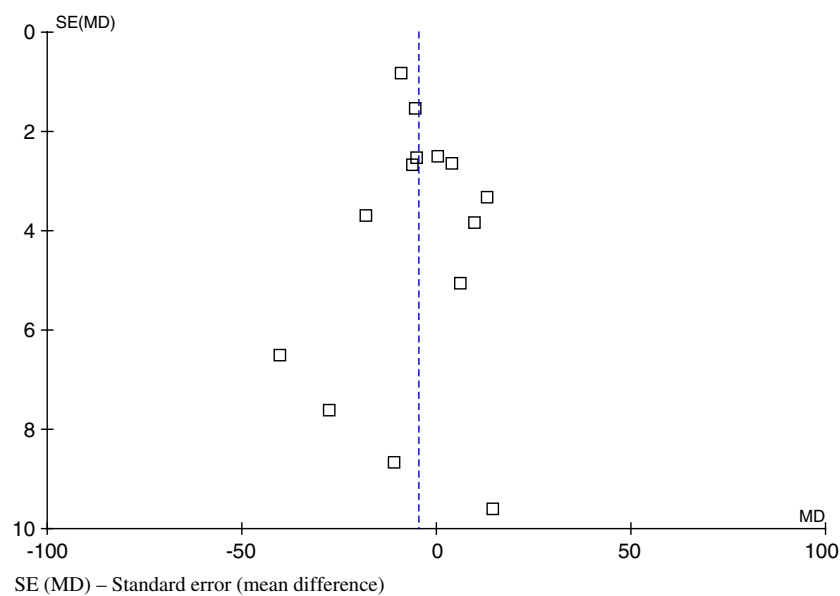

Fig. 2 Funnel plot to assess publication for the most frequently reported outcome - surgical duration. $S E$ (MD) standard error (mean difference) between the groups in respect to drained postoperative blood loss, total blood loss or requirement for blood transfusion ( $p>0.05$; Table 3$)$.

There was no statistically significant difference between the exposure method in respect to WOMAC score $(p=0.13)$ or OHS $(p=0.29)$. Although patients who underwent MIS reported lower pain scores on visual analogue scale (VAS) assessment $(\mathrm{MD}=0.58 ; p=0.02)$ and a shorter hospital length of stay $(\mathrm{MD}=0.59 ; p=0.01)$, these differences were not clinically substantial between the groups.

\section{Radiological outcomes}

As Table 4 demonstrates, there was no statistically significant difference between the MIS or conventional THA exposure methods with respect to any radiological measurement recorded in this meta-analysis.

\section{Complications}

There were no statistically significant differences between the exposure methods during THA for complications such 
Table 1 PEDro critical appraisal score

$Y$ Yes, $N$ No

1. Eligibility criteria

2. Random allocation

3. Concealed allocation

4. Baseline comparability

5. Blind subject

6. Blind clinician

7. Blind assessor

8. Adequate follow-up

9. Intention-to-treat analysis

10. Between-group analysis

11. Point estimates and variability
Study

PEDro criteria

Total

\begin{tabular}{|c|c|c|c|c|c|c|c|c|c|c|c|c|}
\hline & & & & & & & & & & & & \\
\hline & 1 & 2 & 3 & 4 & 5 & 6 & 7 & 8 & 9 & 10 & 11 & \\
\hline Yang et al. [49] & $\mathrm{Y}$ & $\mathrm{Y}$ & $\mathrm{Y}$ & $\mathrm{Y}$ & $\mathrm{Y}$ & $\mathrm{N}$ & $\mathrm{Y}$ & $\mathrm{Y}$ & $\mathrm{N}$ & $\mathrm{Y}$ & $\mathrm{Y}$ & 9 \\
\hline Lawlor et al. [50] & $\mathrm{Y}$ & $\mathrm{Y}$ & $\mathrm{Y}$ & $\mathrm{Y}$ & $\mathrm{Y}$ & $\mathrm{N}$ & $\mathrm{Y}$ & $\mathrm{N}$ & $\mathrm{Y}$ & $\mathrm{Y}$ & $\mathrm{Y}$ & 9 \\
\hline Ogonda et al. [35] & $\mathrm{Y}$ & $\mathrm{Y}$ & $\mathrm{Y}$ & $\mathrm{Y}$ & $\mathrm{Y}$ & $\mathrm{N}$ & $\mathrm{Y}$ & $\mathrm{N}$ & $\mathrm{N}$ & $\mathrm{Y}$ & $\mathrm{Y}$ & 8 \\
\hline Goosen et al. [44] & $\mathrm{Y}$ & $\mathrm{Y}$ & $\mathrm{N}$ & $\mathrm{Y}$ & $\mathrm{N}$ & $\mathrm{N}$ & $\mathrm{Y}$ & $\mathrm{Y}$ & $\mathrm{N}$ & $\mathrm{Y}$ & $\mathrm{Y}$ & 8 \\
\hline Chimento et al. [6] & $\mathrm{Y}$ & $\mathrm{Y}$ & $\mathrm{N}$ & $\mathrm{Y}$ & $\mathrm{N}$ & $\mathrm{N}$ & $\mathrm{Y}$ & $\mathrm{Y}$ & $\mathrm{Y}$ & $\mathrm{Y}$ & $\mathrm{Y}$ & 8 \\
\hline Kim [51] & $\mathrm{Y}$ & $\mathrm{Y}$ & $\mathrm{N}$ & $\mathrm{Y}$ & $\mathrm{N}$ & $\mathrm{N}$ & $\mathrm{Y}$ & $\mathrm{Y}$ & $\mathrm{N}$ & $\mathrm{Y}$ & $\mathrm{Y}$ & 7 \\
\hline Dorr et al. [21] & $\mathrm{Y}$ & $\mathrm{Y}$ & $\mathrm{N}$ & $\mathrm{Y}$ & $\mathrm{N}$ & $\mathrm{N}$ & $\mathrm{Y}$ & $\mathrm{N}$ & $\mathrm{Y}$ & Y & $\mathrm{Y}$ & 7 \\
\hline Bennett et al. [22] & $\mathrm{N}$ & $\mathrm{Y}$ & $\mathrm{Y}$ & $\mathrm{Y}$ & $\mathrm{N}$ & $\mathrm{N}$ & $\mathrm{Y}$ & $\mathrm{N}$ & $\mathrm{Y}$ & $\mathrm{Y}$ & $\mathrm{N}$ & 6 \\
\hline Rittmeister and Peters [52] & $\mathrm{Y}$ & $\mathrm{N}$ & $\mathrm{N}$ & $\mathrm{Y}$ & $\mathrm{N}$ & $\mathrm{N}$ & $\mathrm{N}$ & $\mathrm{N}$ & $\mathrm{Y}$ & $\mathrm{Y}$ & $\mathrm{Y}$ & 5 \\
\hline Leuchte et al. [53] & Y & $\mathrm{N}$ & $\mathrm{N}$ & $\mathrm{N}$ & $\mathrm{N}$ & $\mathrm{N}$ & $\mathrm{N}$ & $\mathrm{N}$ & $\mathrm{Y}$ & Y & $\mathrm{Y}$ & 5 \\
\hline Kubeš et al. [54] & $\mathrm{Y}$ & $\mathrm{N}$ & $\mathrm{N}$ & $\mathrm{Y}$ & $\mathrm{N}$ & $\mathrm{N}$ & $\mathrm{N}$ & $\mathrm{Y}$ & $\mathrm{N}$ & $\mathrm{Y}$ & $\mathrm{Y}$ & 5 \\
\hline Chen et al. [26] & $\mathrm{Y}$ & $\mathrm{N}$ & $\mathrm{N}$ & $\mathrm{N}$ & $\mathrm{N}$ & $\mathrm{N}$ & $\mathrm{N}$ & $\mathrm{Y}$ & $\mathrm{N}$ & Y & $\mathrm{Y}$ & 5 \\
\hline Szendrõi et al. [55] & $\mathrm{Y}$ & $\mathrm{N}$ & $\mathrm{N}$ & $\mathrm{Y}$ & $\mathrm{N}$ & $\mathrm{N}$ & $\mathrm{Y}$ & $\mathrm{N}$ & $\mathrm{N}$ & $\mathrm{Y}$ & $\mathrm{Y}$ & 5 \\
\hline Shitama et al. [20] & $\mathrm{Y}$ & $\mathrm{Y}$ & $\mathrm{N}$ & $\mathrm{Y}$ & $\mathrm{N}$ & $\mathrm{N}$ & $\mathrm{N}$ & $\mathrm{N}$ & $\mathrm{N}$ & $\mathrm{Y}$ & $\mathrm{Y}$ & 5 \\
\hline Sculco et al. [23]-2nd & $\mathrm{N}$ & $\mathrm{Y}$ & $\mathrm{N}$ & $\mathrm{Y}$ & $\mathrm{N}$ & $\mathrm{N}$ & $\mathrm{N}$ & $\mathrm{Y}$ & $\mathrm{N}$ & $\mathrm{Y}$ & $\mathrm{N}$ & 4 \\
\hline Speranza et al. [32] & $\mathrm{Y}$ & $\mathrm{Y}$ & $\mathrm{N}$ & $\mathrm{Y}$ & $\mathrm{N}$ & $\mathrm{N}$ & $\mathrm{N}$ & $\mathrm{N}$ & $\mathrm{N}$ & Y & $\mathrm{N}$ & 4 \\
\hline Vicente et al. [1] & $\mathrm{N}$ & $\mathrm{N}$ & $\mathrm{N}$ & $\mathrm{Y}$ & $\mathrm{N}$ & $\mathrm{N}$ & $\mathrm{N}$ & $\mathrm{N}$ & $\mathrm{Y}$ & $\mathrm{Y}$ & $\mathrm{Y}$ & 4 \\
\hline Wenz et al. [47] & $\mathrm{Y}$ & $\mathrm{N}$ & $\mathrm{N}$ & $\mathrm{N}$ & $\mathrm{N}$ & $\mathrm{N}$ & $\mathrm{N}$ & $\mathrm{N}$ & $\mathrm{Y}$ & $\mathrm{Y}$ & $\mathrm{Y}$ & 4 \\
\hline Pospischill et al. [56] & $\mathrm{Y}$ & $\mathrm{Y}$ & $\mathrm{N}$ & $\mathrm{N}$ & $\mathrm{N}$ & $\mathrm{N}$ & $\mathrm{N}$ & $\mathrm{N}$ & $\mathrm{N}$ & Y & $\mathrm{Y}$ & 4 \\
\hline Mow et al. [48] & $\mathrm{N}$ & $\mathrm{N}$ & $\mathrm{N}$ & $\mathrm{N}$ & $\mathrm{N}$ & $\mathrm{N}$ & $\mathrm{Y}$ & $\mathrm{Y}$ & $\mathrm{Y}$ & $\mathrm{Y}$ & $\mathrm{N}$ & 4 \\
\hline Laffosse et al. [45] & $\mathrm{N}$ & $\mathrm{N}$ & $\mathrm{N}$ & $\mathrm{Y}$ & $\mathrm{N}$ & $\mathrm{N}$ & $\mathrm{N}$ & $\mathrm{N}$ & $\mathrm{Y}$ & $\mathrm{Y}$ & $\mathrm{Y}$ & 4 \\
\hline Laffosse et al. [57] & $\mathrm{Y}$ & $\mathrm{N}$ & $\mathrm{N}$ & $\mathrm{N}$ & $\mathrm{N}$ & $\mathrm{N}$ & $\mathrm{N}$ & $\mathrm{N}$ & $\mathrm{Y}$ & $\mathrm{Y}$ & $\mathrm{Y}$ & 4 \\
\hline Howell et al. [38] & $\mathrm{Y}$ & $\mathrm{N}$ & $\mathrm{N}$ & $\mathrm{N}$ & $\mathrm{N}$ & $\mathrm{N}$ & $\mathrm{N}$ & $\mathrm{N}$ & $\mathrm{Y}$ & $\mathrm{Y}$ & $\mathrm{Y}$ & 4 \\
\hline Wohlrab et al. [58] & $\mathrm{Y}$ & $\mathrm{N}$ & $\mathrm{N}$ & $\mathrm{Y}$ & $\mathrm{N}$ & $\mathrm{N}$ & $\mathrm{N}$ & $\mathrm{N}$ & $\mathrm{N}$ & Y & $\mathrm{N}$ & 3 \\
\hline Woolson et al. [30] & $\mathrm{Y}$ & $\mathrm{N}$ & $\mathrm{N}$ & $\mathrm{N}$ & $\mathrm{N}$ & $\mathrm{N}$ & $\mathrm{Y}$ & $\mathrm{N}$ & $\mathrm{N}$ & Y & $\mathrm{N}$ & 3 \\
\hline Wright et al. [9] & $\mathrm{N}$ & $\mathrm{N}$ & $\mathrm{N}$ & $\mathrm{N}$ & $\mathrm{N}$ & $\mathrm{N}$ & $\mathrm{N}$ & $\mathrm{Y}$ & $\mathrm{N}$ & $\mathrm{Y}$ & $\mathrm{Y}$ & 3 \\
\hline Sculco et al. [23] & $\mathrm{N}$ & $\mathrm{N}$ & $\mathrm{N}$ & $\mathrm{Y}$ & $\mathrm{N}$ & $\mathrm{N}$ & $\mathrm{N}$ & $\mathrm{Y}$ & $\mathrm{N}$ & $\mathrm{Y}$ & $\mathrm{N}$ & 3 \\
\hline Pflüger et al. [59] & $\mathrm{N}$ & $\mathrm{N}$ & $\mathrm{N}$ & $\mathrm{N}$ & $\mathrm{N}$ & $\mathrm{N}$ & $\mathrm{N}$ & $\mathrm{N}$ & $\mathrm{Y}$ & $\mathrm{Y}$ & $\mathrm{N}$ & 2 \\
\hline
\end{tabular}

as infection rates, intra- or postoperative fracture, dislocation rate, DVT, haematoma formation, wound complications or component loosening. There was however a statically significant difference in respect to iatrogenic nerve palsy with a five times greater rate of nerve palsy following MIS surgery compared to conventional THA $(p<0.0001$; Fig. 4$)$. When assessed individually, the risk of transient lateral femoral cutaneous nerve palsy was significantly higher following MIS (RR=16.2; $p=0.006$; Fig. 5); however, this finding was weighted by a high proportion of cases reported in a cohort study by Chen et al. [26]. There was no statistically significant difference between the groups with respect to the incidence of sciatic nerve palsy $(p=0.11$; Table 5).

\section{Discussion}

The findings of this review of the current evidence base suggest that MIS THA results in a significantly increased risk of lateral femoral cutaneous nerve palsy. There was no clinically significant reduction in total blood loss or hip scores at final follow-up with no difference in radiological outcomes at final review compared to a conventional approach. Whilst hospital stay and pain scores were lower in the MIS group, this was not a clinically significant difference.

The PEDro appraisal identified a number of methodological limitations to the current evidence base. These were largely cited as poor concealment of randomisation, permitting selection and allocation bias, not blinding patients and assessors to their surgical exposure, allowing further expectation and assessor bias, and not recruiting sample sizes based on an appropriate power calculation, allowing the potential for type II statistical error from impacting on the findings of these clinical studies [27, 28]. Accordingly, whilst the findings of this meta-analysis should be considered as appropriate, based on the best available literature, these methodological shortcomings should be considered when interpreting the findings. 
Table 2 Cohort characteristics

\begin{tabular}{|c|c|c|c|c|c|c|c|c|c|c|c|}
\hline \multirow[t]{2}{*}{ Study } & \multirow[t]{2}{*}{ Design } & \multicolumn{2}{|c|}{$\begin{array}{l}\text { Sample } \\
\text { Size }\end{array}$} & \multicolumn{2}{|l|}{ THA } & \multicolumn{2}{|c|}{$\begin{array}{l}\text { Mean age } \\
\text { (years) }\end{array}$} & \multicolumn{2}{|c|}{$\begin{array}{l}\text { Gender } \\
(\mathrm{m} / \mathrm{f})\end{array}$} & \multirow[t]{2}{*}{ Approach } & \multirow[t]{2}{*}{ Follow-up } \\
\hline & & Pts & THAs & MIS & Conv & MIS & Conv & MIS & Conv & & \\
\hline Bennett et al. [22] & $\mathrm{RCT}$ & 95 & 95 & 43 & 52 & 66.1 & 64.6 & $18 / 25$ & $28 / 24$ & $\begin{array}{l}\text { MIS-mini-posterior }(\leq 10 \mathrm{~cm}) \\
\text { Conv-std posterior }(16 \mathrm{~cm})\end{array}$ & 5 days \\
\hline Chen et al. [26] & $\mathrm{nRCT}$ & 166 & 166 & 83 & 83 & 53.5 & 55 & $46 / 37$ & $41 / 42$ & $\begin{array}{l}\text { MIS-mini anterolateral } \\
\text { Conv-std anterolateral }\end{array}$ & 2 years \\
\hline Chimento et al. [6] & $\mathrm{RCT}$ & 60 & 60 & 28 & 32 & 67.2 & 65.6 & $16 / 12$ & $13 / 19$ & $\begin{array}{l}\text { MIS-mini posterolateral } \\
\text { Conv-std posterolateral }\end{array}$ & 2 years \\
\hline Dorr et al. [21] & $\mathrm{RCT}$ & 60 & 60 & 30 & 30 & 70.3 & 63.9 & $17 / 13$ & $14 / 16$ & $\begin{array}{l}\text { MIS-Mini-posterior with } \\
\text { navigation in } 27 / 30 \\
\text { Conv-Posterior }\end{array}$ & 6 months \\
\hline Goosen et al. [44] & RCT & 120 & 120 & 60 & 60 & 60 & 62 & $30 / 30$ & $29 / 31$ & $\begin{array}{l}\text { MIS-mini-anterolateral/mini } \\
\text { posterior } \\
\text { Conv-std anterolateral/ } \\
\text { posterior }\end{array}$ & 1 year \\
\hline Howell et al. [38] & $\mathrm{nRCT}$ & 107 & 107 & 50 & 57 & 59.8 & 62.3 & $34 / 16$ & $27 / 30$ & $\begin{array}{l}\text { MIS-mini-anterolateral } \\
\text { Conv-std anterolateral }\end{array}$ & $\mathrm{N} / \mathrm{S}$ \\
\hline Kim [51] & $\mathrm{RCT}$ & 140 & 140 & 70 & 70 & 55.6 & 55.6 & $53 / 17$ & $53 / 17$ & $\begin{array}{l}\text { MIS-mini-posterolateral } \\
\text { Conv-std posterolateral }\end{array}$ & 26.4 months \\
\hline Kubeš et al. [54] & $\mathrm{nRCT}$ & 80 & 80 & 40 & 40 & 67 & 66.1 & $14 / 26$ & $19 / 26$ & $\begin{array}{l}\text { MIS-mini anterolateral } \\
\text { Conv-std anterolateral }\end{array}$ & 2 years \\
\hline Laffosse et al. [45] & $\mathrm{nRCT}$ & 100 & 100 & 42 & 58 & 57.4 & 59.7 & $24 / 18$ & $33 / 25$ & $\begin{array}{l}\text { MIS-mini-anterolateral } \\
\text { Conv-std posterior }\end{array}$ & 6 months \\
\hline Laffosse et al. [57] & $\mathrm{nRCT}$ & 110 & 116 & 58 & 58 & 55 & 59.7 & $35 / 23$ & $33 / 25$ & $\begin{array}{l}\text { MIS-mini-posterior } \\
\text { Conv-std posterolateral }\end{array}$ & 6 months \\
\hline Lawlor et al. [50] & $\mathrm{RCT}$ & 219 & 219 & 109 & 110 & 67.4 & 65.9 & $49 / 60$ & $58 / 52$ & $\begin{array}{l}\text { MIS-mini-posterior } \leq 10 \mathrm{~cm} \\
\text { Conv-std posterior } 16 \mathrm{~cm}\end{array}$ & 6 weeks \\
\hline Leuchte et al. [53] & $\mathrm{nRCT}$ & 32 & 32 & 16 & 16 & 59.7 & 62.6 & $\mathrm{~N} / \mathrm{S}$ & $\mathrm{N} / \mathrm{S}$ & $\begin{array}{l}\text { MIS-mini anterolateral } \\
\text { Conv-std lateral }\end{array}$ & 28 weeks \\
\hline Mow et al. [48] & $\mathrm{nRCT}$ & 32 & 34 & 20 & 14 & 59 & 63 & $13 / 6$ & $7 / 6$ & $\begin{array}{l}\text { MIS-mini-posterior } \\
\text { Conv-direct lateral }\end{array}$ & 24 months \\
\hline Ogonda et al. [35] & $\mathrm{RCT}$ & 219 & 219 & 109 & 110 & 67.4 & 65.9 & $49 / 60$ & $58 / 52$ & $\begin{array}{l}\text { MIS-mini-posterior } \leq 10 \mathrm{~cm} \\
\text { Conv-std posterior } 16 \mathrm{~cm}\end{array}$ & 6 weeks \\
\hline Pflüger et al. [59] & $\mathrm{nRCT}$ & 100 & 100 & 50 & 50 & $\mathrm{~N} / \mathrm{S}$ & $\mathrm{N} / \mathrm{S}$ & $\mathrm{N} / \mathrm{S}$ & $\mathrm{N} / \mathrm{S}$ & $\begin{array}{l}\text { MIS-mini anterolateral } \\
\text { Conv-std anterolateral }\end{array}$ & $\mathrm{N} / \mathrm{S}$ \\
\hline Pospischill et al. [56] & $\mathrm{RCT}$ & 40 & 40 & 20 & 20 & 61.9 & 60.6 & $8 / 12$ & $12 / 8$ & $\begin{array}{l}\text { MIS-mini-anterolateral } \\
\text { approach } \\
\text { Conv-std lateral approach }\end{array}$ & 12 weeks \\
\hline Rittmeister and Peters [52] & $\mathrm{nRCT}$ & 152 & 152 & 76 & 76 & 60 & 65 & $23 / 53$ & $23 / 53$ & $\begin{array}{l}\text { MIS-mini-posterior } \\
\text { Conv-anterolateral }\end{array}$ & 4 days \\
\hline Sculco et al. [23] & $\mathrm{nRCT}$ & 84 & 84 & 42 & 42 & 67.2 & 65.6 & $12 / 16$ & $19 / 13$ & $\begin{array}{l}\text { MIS-mini-anterolateral } \\
\text { Conv-N/S }\end{array}$ & 5 years \\
\hline Sculco et al. [23] - 2nd study & $\mathrm{RCT}$ & 60 & 60 & 28 & 32 & $\mathrm{~N} / \mathrm{S}$ & $\mathrm{N} / \mathrm{S}$ & $\mathrm{N} / \mathrm{S}$ & $\mathrm{N} / \mathrm{S}$ & $\begin{array}{l}\text { MIS-mini-anterolateral } \\
(8 \mathrm{~cm}) \\
\text { Conv-N/S (15 cm incision })\end{array}$ & Min 2 years \\
\hline Shitama et al. PL [20] & RCT & 39 & 39 & 19 & 20 & 58.3 & 61.3 & $\mathrm{~N} / \mathrm{S}$ & $\mathrm{N} / \mathrm{S}$ & $\begin{array}{l}\text { MIS-mini-posterolateral } \\
\text { Conv-posterolateral }\end{array}$ & 6 months \\
\hline Shitama et al. TL [20] & $\mathrm{RCT}$ & 23 & 23 & 15 & 8 & 61.7 & 53.4 & $\mathrm{~N} / \mathrm{S}$ & $\mathrm{N} / \mathrm{S}$ & $\begin{array}{l}\text { MIS-mini-translateral } \\
\text { Conv-translateral }\end{array}$ & 6 months \\
\hline Speranza et al. [30] & $\mathrm{RCT}$ & 100 & 100 & 50 & 50 & 65 & 66.2 & $20 / 26$ & $23 / 21$ & $\begin{array}{l}\text { MIS-mini-lateral }(\leq 8 \mathrm{~cm}) \\
\text { Conv-std lateral }(12-14 \mathrm{~cm})\end{array}$ & 6 months \\
\hline Szemdrõi et al. [55] & $\mathrm{nRCT}$ & 59 & 59 & 38 & 21 & 64 & 57 & $\mathrm{~N} / \mathrm{S}$ & $\mathrm{N} / \mathrm{S}$ & $\begin{array}{l}\text { MIS-mini-lateral }(<10 \mathrm{~cm}) \\
\text { Conv-std lateral }(>14 \mathrm{~cm})\end{array}$ & 3 months \\
\hline Vicente et al. [1] & $\mathrm{nRCT}$ & 76 & 76 & 34 & 42 & 50 & 57 & $21 / 13$ & $26 / 16$ & MIS-mini posterior $<11 \mathrm{~cm}$ & 6 months \\
\hline
\end{tabular}


Table 2 (continued)

\begin{tabular}{|c|c|c|c|c|c|c|c|c|c|c|c|}
\hline \multirow[t]{2}{*}{ Study } & \multirow[t]{2}{*}{ Design } & \multicolumn{2}{|c|}{$\begin{array}{l}\text { Sample } \\
\text { Size }\end{array}$} & \multicolumn{2}{|l|}{ THA } & \multicolumn{2}{|c|}{$\begin{array}{l}\text { Mean age } \\
\text { (years) }\end{array}$} & \multicolumn{2}{|c|}{$\begin{array}{l}\text { Gender } \\
(\mathrm{m} / \mathrm{f})\end{array}$} & \multirow[t]{2}{*}{ Approach } & \multirow[t]{2}{*}{ Follow-up } \\
\hline & & Pts & THAs & MIS & Conv & MIS & Conv & MIS & Conv & & \\
\hline Wenz et al. [47] & $\mathrm{nRCT}$ & 173 & 189 & 124 & 65 & 63 & 65 & $60 / 64$ & $22 / 43$ & $\begin{array}{l}\text { Conv-direct lateral } \\
\text { MIS-mini-posterior } \\
\text { Conv-direct lateral }\end{array}$ & $\mathrm{N} / \mathrm{S}$ \\
\hline Wohlrab et al. [58] & $\mathrm{nRCT}$ & 50 & 50 & 27 & 23 & 58.8 & 61.9 & $11 / 26$ & $11 / 12$ & $\begin{array}{l}\text { MIS-mini posterior } \\
\text { Conv-std lateral }\end{array}$ & 3 months \\
\hline Woolson et al. [30] & $\mathrm{nRCT}$ & 135 & 135 & 50 & 85 & 60 & 63 & $29 / 21$ & $31 / 54$ & $\begin{array}{l}\text { MIS-mini-posterior } \\
\text { Conv-posterior }\end{array}$ & Min 6 months \\
\hline Wright et al. [9] & $\mathrm{nRCT}$ & 84 & 84 & 42 & 42 & 65.0 & 64.2 & $\mathrm{~N} / \mathrm{S}$ & $\mathrm{N} / \mathrm{S}$ & $\begin{array}{l}\text { MIS-mini-posterolateral } \\
\text { Conv-posterolateral }\end{array}$ & 5 years \\
\hline Yang et al. [49] & $\mathrm{RCT}$ & 110 & 110 & 55 & 55 & 59 & 56 & $26 / 29$ & $30 / 25$ & $\begin{array}{l}\text { MIS-mini-anterolateral } \\
\text { Conv-posterolateral }\end{array}$ & 3 years \\
\hline
\end{tabular}

THA total hip arthroplasty, Conv conventional surgery, $f$ females, $m$ males, Min minimum, MIS minimally invasive surgery, $n R C T$ non-randomised controlled trial, $N / S$ not stated, $P L$ posterolateral approach, $R C T$ randomised controlled trial, $T L$ translateral approach

A major finding reported by the overall meta-analysis was the significantly greater risk of iatrogenic nerve injury during MIS compared to conventional procedures. One suggestion for this is related to retractor position. Yoon et al. [29] suggested that femoral nerve palsy, for instance, may be associated with retractor position [29]. The anterior retractor should be underneath the rectus femoris muscle to prevent this. Similarly, reduced operative visibility may increase the potential for nerve injury due to the added difficulty in identifying nerves during dissection.

Although not included in this meta-analysis, Woolson et al. [30] reported acetabular and femoral prostheses were

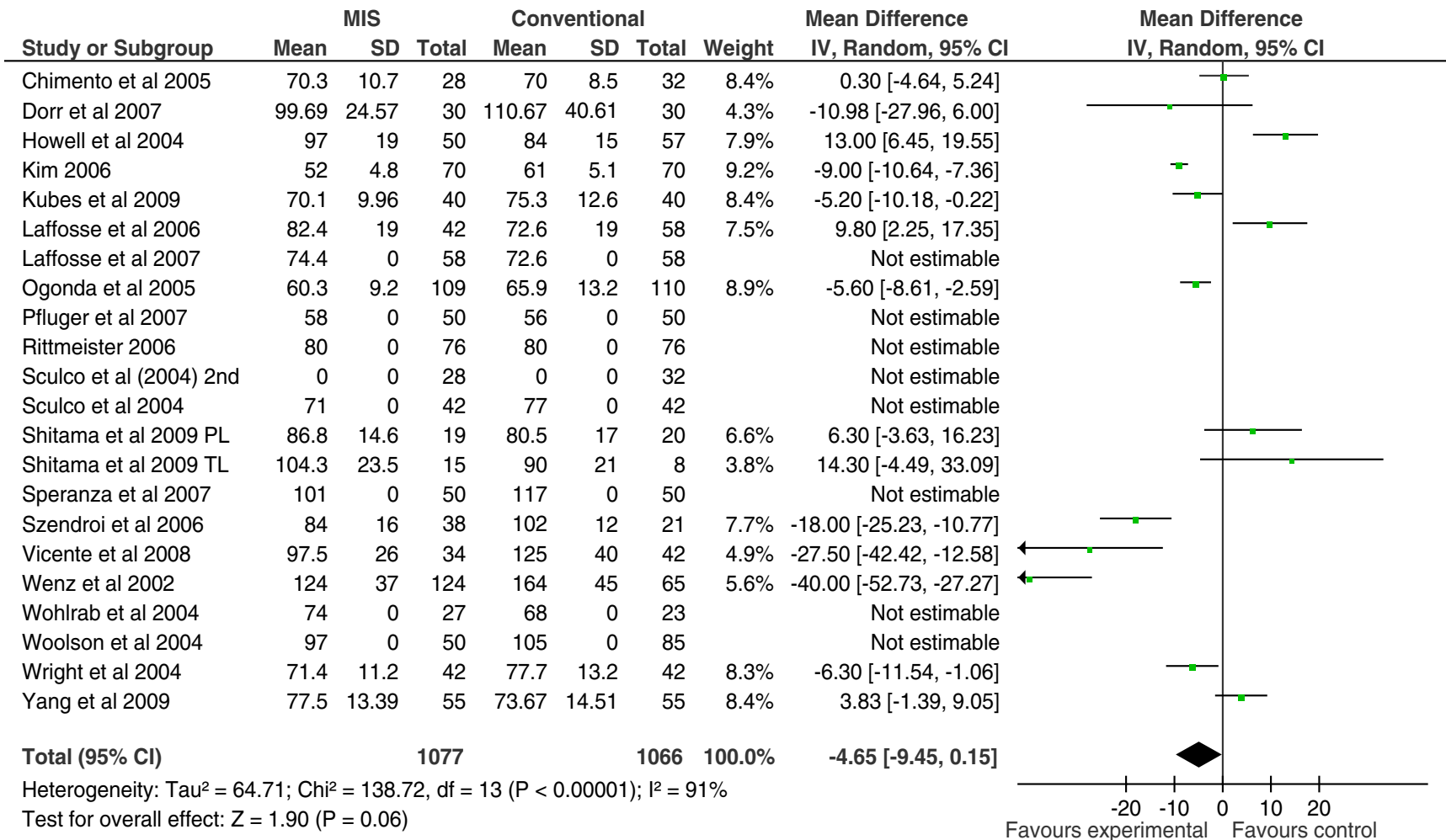

Fig. 3 Forest plot to illustrate mean difference in Harris hips score between minimally invasive surgery (MIS) and conventional total hip arthroplasty (THA) procedures 
Table 3 Meta-analysis results of clinical outcomes

\begin{tabular}{|c|c|c|c|c|c|c|}
\hline \multirow[t]{2}{*}{ Outcome } & \multicolumn{2}{|c|}{ Groups $(n)$} & \multirow[t]{2}{*}{ Studies $(n)$} & \multicolumn{3}{|l|}{ Overall effect } \\
\hline & MIS & Conv & & Effect estimate & $95 \% \mathrm{CI}$ & $p$-value \\
\hline Incision length & 869 & 803 & 17 & -7.56 & $-8.17,-6.95$ & $<0.0001$ \\
\hline Intraoperative blood loss & 621 & 656 & 12 & -42.44 & $-60.14,-24.73$ & $<0.0001$ \\
\hline Length of stay & 840 & 856 & 15 & -0.59 & $-1.07,-0.12$ & 0.01 \\
\hline VAS pain ${ }^{\mathrm{a}}$ & 359 & 339 & 7 & -0.55 & $-0.97,-0.13$ & 0.01 \\
\hline Harris hip score & 784 & 797 & 17 & 1.49 & $0.08,3.06$ & 0.06 \\
\hline Surgical duration & 1077 & 1066 & 22 & -4.65 & $-9.45,0.15$ & 0.06 \\
\hline Required blood transfusion & 95 & 133 & 7 & 0.75 & $-0.56,1.02$ & 0.06 \\
\hline WOMAC score & 402 & 419 & 6 & 2.55 & $-0.75,5.84$ & 0.13 \\
\hline Blood loss in drain & 200 & 195 & 6 & -53.46 & $-133.55,26.62$ & 0.19 \\
\hline Oxford hip score & 169 & 170 & 2 & -0.92 & $-2.62,0.77$ & 0.29 \\
\hline Total blood loss & 738 & 701 & 15 & -43.09 & $-135.79,49.62$ & 0.36 \\
\hline
\end{tabular}

MIS minimally invasive surgery, Conv conventional surgery, $C I$ confidence interval

${ }^{\mathrm{a}}$ Standardised mean difference

more frequently mal-positioned in MIS compared to conventional approaches. Similarly, they reported a significantly higher percentage of cementless stems in the MIS cohort had a poor fit and fill with less than $2 \mathrm{~mm}$ between the distal portion of the stem and the femoral cortex $(p=$ $0.004)$. They related this to the reduced visualisation of the acetabulum and proximal femur from the small incision. Given the findings of this study, such issues in implant positioning do not seem to be supported by the literature. Nonetheless, since we were unable to distinguish the results between experienced and in-experienced MIS surgeons, it remains unclear whether this factor was important when generalising this complication to general clinical practice. Furthermore, since the longest follow-up period documented was five years $[9,23]$, it remains unclear whether the affect of implant positioning has any longer-term effect on prosthesis survival. Future surveillance studies of longer follow-up will enlighten as to whether this is a potential feature of MIS THA procedures.
Although this meta-analysis reported no statistically significant difference between surgical exposure method and wound healing complications $(p=0.17)$, the effect size was substantial between the groups with nearly a three times greater risk following MIS compared to conventional THA in the overall analysis. As Table 5 demonstrates, this outcome was measured in a small number of subjects. Accordingly, this conclusion may be attributed to type II statistical error [28]. Such an effect size for this outcome may be attributed to the extensive use of retractors in MIS procedures. Noble et al. [31] reported that during MIS THA, large pressures can develop between the retractors and the wound edges, predisposing to wound healing complications. They recommended that surgeons should consider using the largest possible incision within the realms of MIS principles, and that the precise anatomical placement should be carefully considered to minimise the duration of tissue compression whilst ensuring adequate visualisation of the surgical field [31].

Table 4 Meta-analysis results of radiological outcomes

\begin{tabular}{|c|c|c|c|c|c|c|}
\hline \multirow[t]{2}{*}{ Outcome } & \multicolumn{2}{|c|}{ Groups $(n)$} & \multirow[t]{2}{*}{ Studies $(n)$} & \multicolumn{3}{|l|}{ Overall effect } \\
\hline & MIS & Conv & & Effect estimate & $95 \% \mathrm{CI}$ & $p$-value \\
\hline Femoral offset & 150 & 150 & 3 & 0.62 & $-0.77,2.01$ & 0.38 \\
\hline Leg-length discrepancy & 325 & 300 & 5 & -0.09 & $-0.32,0.43$ & 0.42 \\
\hline Femoral positioning $\left(0-5^{\circ}\right.$ valgus $)$ & 258 & 177 & 4 & 0.57 & $0.11,2.85$ & 0.49 \\
\hline Cup positioning ( $35-50^{\circ}$ valgus) & 266 & 311 & 5 & 0.82 & $0.42,1.59$ & 0.55 \\
\hline Cup inclination angle & 750 & 730 & 12 & 0.24 & $-1.51,2.00$ & 0.79 \\
\hline Stem alignment (varus/valgus) & 314 & 331 & 5 & 0.03 & $-0.38,0.43$ & 0.90 \\
\hline
\end{tabular}

MIS minimally invasive surgery, Conv conventional surgery, $C I$ confidence interval 


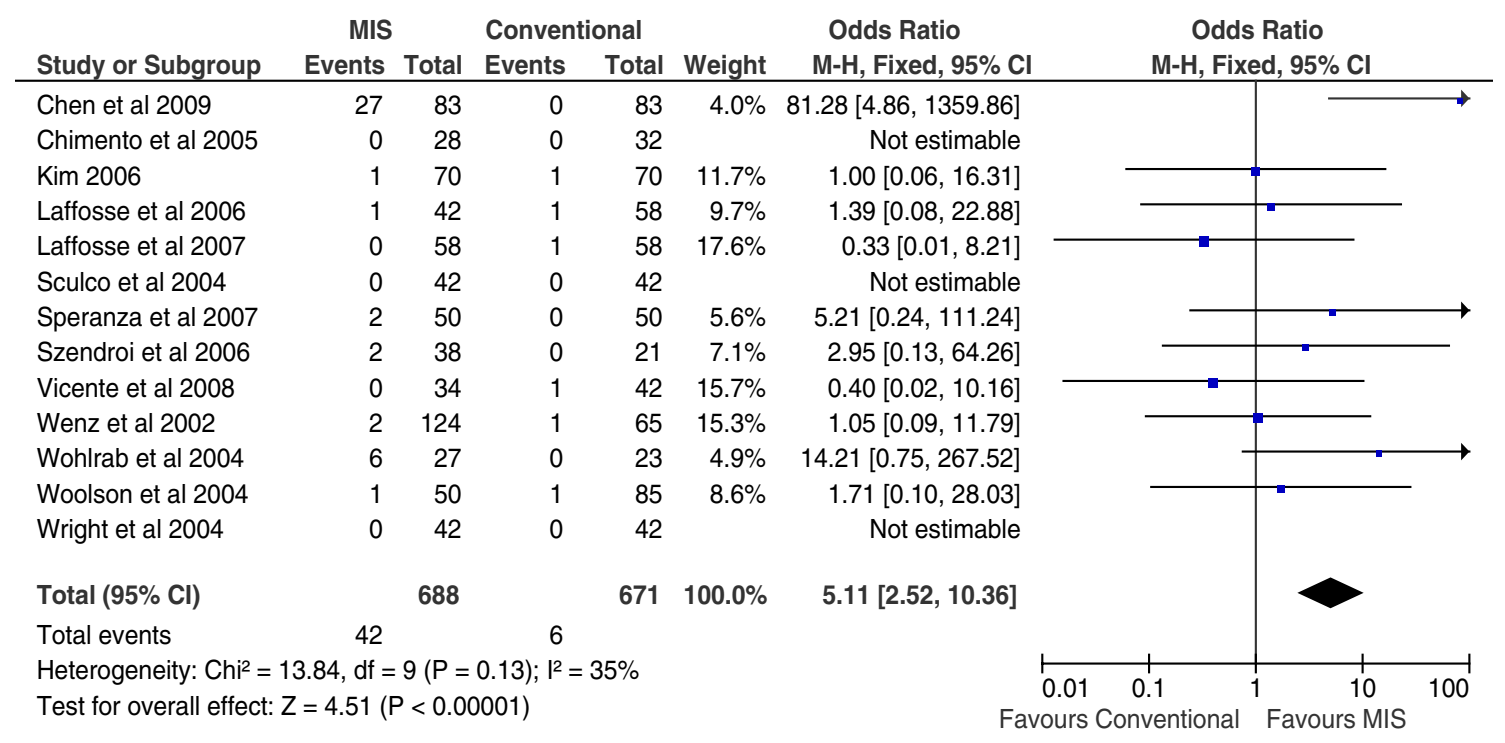

Fig. 4 Forest plot to illustrate odds ratio for incidence of iatrogenic nerve injury between minimally invasive surgical (MIS) and conventional total hip arthroplasty (THA) procedures

There is a lack of consensus over the actual definition of MIS and the relationship between skin incision and soft tissue trauma. Speranza et al. [32] and Procyk [33] suggested that the ideal MIS is that of a procedure which has little tissue disruption without cutting muscles and tendons and therefore less pain to provide a significantly shorter rehabilitation with longer-term outcomes which are equal or better to a conventional approach. Accordingly, the little difference in outcomes reported in this metaanalysis may be attributed to similarities in the operative procedure after the skin incision for traditional and MIS procedures.

An increase in perioperative cytokine level has been demonstrated to correlate with surgical trauma [34]. Both Ogonda et al. [35] and Chimento et al. [6] reported no significant difference between the minimally invasive and conventional THA exposure for cytokine level suggesting that whilst the skin incision may be reduced, tissue trauma is similar between the groups for this procedure. Nonetheless, these findings may however be dependent on the surgical approach adopted and the degree of soft tissue dissection. For instance, in a mini-posterior if the femoral head can be excised without dislocating the hip, there will be less trauma to muscles and capsule unlike the anterolateral approach [36, 37]. Further assessment of surgical approach and dissection is therefore warranted.

Whilst we did not assess the difference in outcomes between the different types of MIS, as Table 2 demonstrated a number of different surgical approaches were adopted under the term 'minimally invasive'. Further study is required to determine whether there is a difference in outcomes between the different MIS procedures used during THA. Nonetheless, theoretically, this factor may be associated with different outcomes and, in particular, the incidence of iatrogenic nerve injury. The anterior approach (as part of the Smith-Peterson approach) may be associated with lateral femoral cutaneous nerve injury which was 17 times more likely to occur with MIS compared to standard incision surgery [29]. Given this important complication, further study is recommended to compare the outcomes of different MIS procedures undertaken in THA surgery to determine the efficacy of each approach taken.

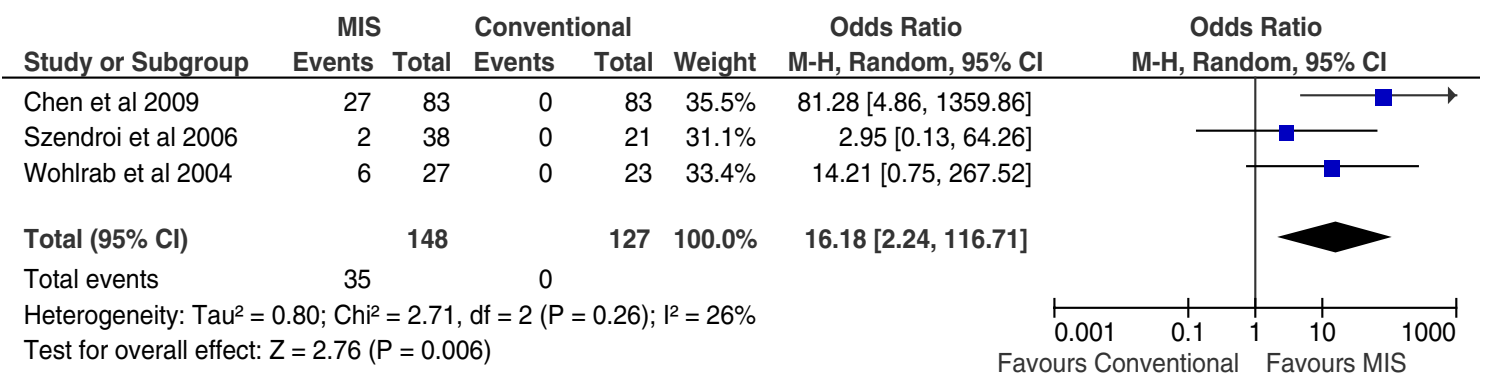

Fig. 5 Forest plot to illustrate odds ratio for incidence of transient lateral femoral cutaneous nerve injury between minimally invasive surgical (MIS) and conventional total hip arthroplasty (THA) procedures 
Table 5 Meta-analysis results of complications

\begin{tabular}{|c|c|c|c|c|c|c|}
\hline \multirow[t]{2}{*}{ Outcome } & \multicolumn{2}{|c|}{ Groups $(n)$} & \multirow[t]{2}{*}{ Studies $(n)$} & \multicolumn{3}{|l|}{ Overall effect } \\
\hline & MIS & Conv & & Effect estimate & $95 \% \mathrm{CI}$ & $p$-value \\
\hline Iatrogenic nerve palsy & 650 & 650 & 13 & 5.27 & $2.55,10.91$ & $<0.0001$ \\
\hline Transient lateral femoral cutaneous nerve injury & 148 & 127 & 3 & 16.18 & $2.24,116.71$ & 0.006 \\
\hline Haematoma formation & 270 & 201 & 4 & 2.48 & $0.85,7.21$ & 0.09 \\
\hline Sciatic nerve palsy & 160 & 195 & 3 & 4.38 & $0.70,27.20$ & 0.11 \\
\hline Wound complication & 112 & 141 & 3 & 2.99 & $0.62,14.35$ & 0.17 \\
\hline Deep vein thrombosis & 529 & 509 & 9 & 0.49 & $0.18,1.35$ & 0.17 \\
\hline Dislocation & 929 & 918 & 16 & 0.65 & $0.33,1.26$ & 0.20 \\
\hline Intraoperative fracture & 563 & 568 & 10 & 1.52 & $0.76,3.04$ & 0.23 \\
\hline Heterotrophic ossification & 85 & 85 & 2 & 0.32 & $0.05,2.10$ & 0.24 \\
\hline Acetabular component loosening & 316 & 324 & 6 & 2.40 & $0.52,10.98$ & 0.26 \\
\hline Periprosthetic fracture & 212 & 223 & 4 & 0.67 & $0.27,1.69$ & 0.40 \\
\hline Required revision surgery & 230 & 250 & 5 & 0.72 & $0.15,3.50$ & 0.68 \\
\hline Deep infection & 594 & 540 & 9 & 0.82 & $0.22,2.97$ & 0.76 \\
\hline Superficial wound infection & 620 & 609 & 10 & 11.08 & $0.35,3.37$ & 0.89 \\
\hline Femoral component loosening & 222 & 222 & NE & $\mathrm{NE}$ & $\mathrm{NE}$ & $\mathrm{NE}$ \\
\hline
\end{tabular}

$M I S$ minimally invasive surgery, Conv conventional surgery, $C I$ confidence interval, $N E$ not estimatable

Surgical learning curve is an important variable which may have accounted for the differences in surgical duration between the groups [30, 38-40]. Desser et al. [41] and Pagnano et al. [42] reported that the MIS technique was more difficult than the conventional exposure method, but that complication rates would be expected to decrease with surgical experience [41, 43]. Whilst some authors have reported a low complication rate such as Berry et al. [13] of $2 \%$ with four experienced surgeons, others have reported much higher rates such as Pagnano et al. [42] conversely with $14 \%$, which was attributed to surgical experience and the existence of a learning curve [13, 43]. Goosen et al. [44] concluded that relatively inexperienced surgeons should consider carefully the advantages and disadvantages of MIS procedures before adopting such an approach given the long learning curve. Furthermore, Sculco et al. [23] suggested that the posterior approach may be the most appropriate approach to adopt since it is familiar to most surgeons and still allows the easy extension of the wound if operative visibility is insufficient $[23,45]$.

When considering its application in clinical practice, previous authors have suggested that not all patients are suitable candidates for MIS THA procedures [41]. Those patients who are thin and young, and with a lower risk of peri- or postoperative complication would be most suitable for MIS [46]. However, as Desser et al. [41] commented, these characteristics may not be reflective of the average hip surgeon's caseload. Sculco et al. [23] suggested that patients with a body mass index greater than 30 should not be considered for MIS due to the difficulty in identifying anatomical landmarks during surgery. Howell et al. [38] also suggested that patients with excessively stiff hips, those with severe dysplasia requiring larger visualisation to manage the distorted acetabular anatomy and correct any femoral shortening or derotation, and those with a marked distortion of the proximal femur may also be unsuitable for MIS THA. For such larger patients a secondary incision may be required distally to allow adequate acetabular reaming [47].

Advocates of the MIS technique have suggested that the smaller skin incision provides improved cosmesis and increased patient satisfaction. Only one study has previously assessed the outcomes of scar cosmesis following conventional or MIS THA [48]. Whilst all patients considered their scars acceptable in appearance, when reviewed by plastic surgeons masked to surgical procedure, the cosmesis of the mini-posterior approach was more frequently reported as poorer than the standard posterior approach. Mow et al. [48] attribute these findings to skin and soft tissue damage caused by the high retractor pressure required for the MIS exposure.

\section{Conclusions}

The findings of this study indicate whilst there is little difference in the clinical or radiological outcomes of MIS to conventional THA, MIS procedures pose a significantly increased risk of transient lateral femoral cutaneous nerve palsy than traditional techniques. 
Acknowledgements We thank the library staff at the Norfolk and Norwich University Hospital's Sir Thomas Browne Library for their assistance in gathering the articles used in this review. We would also like to thank Professor Young-Hoo Kim, MokDong Hospital, Seoul, Korea; Dr J Ricardo Negreiros Vicente, Faculdade de Medicina da Universidade de São Paulo, São Paulo, Brazil; Dr Thomas P. Sculco, Hospital for Special Surgery, New York, USA; Professor Qingsheng Zhu, Xijing Hospital, China; Marie Lawlor, Musgrave Park Hospital, Belfast, Northern Ireland; Mr Lawrence Dorr, Arthritis Institute, Inglewood, California, USA; and Professor Steve Woolson, Stanford University Hospital, Stanford, California, USA, for providing additional data used as part of the meta-analysis and for reviewing the results of our search strategy.

Conflict of interest The authors declare that they have no conflict of interest

Funding No funds were received to undertake or relating to this study.

Ethical approval Ethical approval was not required for this study design.

\section{References}

1. Vicente JRN, Croci AT, Camargo OP (2008) Blood loss in the minimally invasive posterior approach to total hip arthroplasty: a comparative study. Clinics 63:351-356

2. Moore AT (1952) Metal hip joint; a new self-locking vitallium prosthesis. S Med J 45:1015-1019

3. Hardinge K (1982) The direct lateral approach to the hip. J Bone Joint Surg 64-B:17-19

4. Beger RA, Jacobs JJ, Meneghini RM et al (2004) Rapid rehabilitation and recovery with minimally invasive total hip arthroplatsty. Clin Orthop Relat Res 429:239-247

5. Goldstein WM, Bransson JJ, Berland KA, Gordon AC (2003) Minimal-incision total hip arthroplasty. J Bone Joint Surg 85-A (Suppl 4):33-38

6. Chimento GF, Pavone V, Sharrock N, Kahn B, Cahill J, Sculco TP (2005) Minimally invasive total hip arthroplasty: a prospective randomised study. J Arthroplasty 20:139-144

7. Inaba Y, Dorr LD, Wan Z, Sirianni L, Boutary M (2005) Operative and patient care techniques for posterior miniincision total hip arthroplasty. Clin Orthop Relat Res 441:104-114

8. Dorr LD, Long WT, Inaba Y, Sirianni LE, Boutary M (2005) MIS total hip replacement with a single posterior approach. Semin Arthroplasty 16:179-185

9. Wright JM, Crockett HC, Delgado S et al (2004) Mini-incision for total hip arthroplasty. A prospective, controlled investigation with 5-year follow-up evaluation. J Arthroplasty 19:538545

10. Nakamura S, Matsuda K, Arai N, Wakimoto N, Matsushita T (2004) Mini-incision posterior approach for total hip arthroplasty. Int Orthop 28:214-217

11. Hartzband MA (2004) Posterolateral minimal incision for total hip replacement: technique and early results. Orthop Clin N Am 34:119-129

12. O'Brien DA, Rorabeck CH (2005) The mini-incision direct lateral approach in primary total hip arthroplasty. Clin Orthop Relat Res 441:99-103
13. Berry DJ, Berger RA, Callaghan JJ et al (2003) Minimally invasive total hip arthroplasty. Development, early results and a critical analysis. J Bone Joint Surg 85-A:2235-2246

14. Mardones R, Pagnano MW, Nemanich JP, Trousdale RT (2005) Muscle damage after total hip arthroplasty done with the twoincision and mini-posterior techniques. Clin Orthop Relat Res 441:80-85

15. Cheng T, Feng JG, Liu T, Zhang XL (2009) Minimally invasive total hip arthroplasty: a systematic review. Int Orthop 33:14731481

16. Foley NC, Bhogal SK, Teasell RW, Bureau Y, Speechley MR (2006) Estimates of quality and reliability with the physiotherapy evidence-based database scale to assess the methodology of randomized controlled trials of pharmacological and nonpharmacological interventions. Phys Ther 86:817-824

17. Maher CG, Sherrington C, Herbert RD, Moseley AM, Elkins M (2003) Reliability of the PEDro scale for rating quality of randomized controlled trials. Phys Ther 83:713-721

18. Mantel N, Haenszel W (1959) Statistical aspects of the analysis of data from retrospective studies of disease. J Natl Cancer Inst 22:719-748

19. Higgins JPT, Thompson SG, Deeks JJ, Altman DG (2003) Measuring inconsistency in meta-analyses. BMJ 327:557-560

20. Shitama T, Kiyama T, Naito M, Shiramizu K, Huang G (2009) Which is more invasive-mini versus standard incisions in total hip arthroplasty? Int Orthop 33:1543-1547

21. Dorr LD, Maheshwari AV, Long WT, Wan Z, Sirianni LE (2007) Early pain relief and function after posterior minimally invasive and conventional total hip arthroplasty. A prospective, randomized, blinded study. J Bone Joint Surg 89:1153-1160

22. Bennett D, Ogonda L, Elliott D, Humphreys L, Lawlor M, Beverland D (2007) Comparison of immediate postoperative walking ability in patients receiving minimally invasive and standard-incision hip arthroplasty. A prospective blinded study. J Arthroplasty 22:490-495

23. Sculco TP, Jordan LC, Walter WL (2004) Minimally invasive total hip arthroplasty: the Hospital for Special Surgery experience. Orthop Clin N Am 35:137-142

24. Harris WH (1969) Traumatic arthritis of the hip after dislocation and acetabular fractures: treatment by mold arthroplasty. An endresult study using a new method of result evaluation. J Bone Joint Surg 51-A:737-755

25. Marchetti P, Binazzi R, Vaccari V et al (2005) Long-term results with cementless Fitek (or Fitmore) cups. J Arthroplasty 20:730 737

26. Chen DW, Hu CC, Chang YH, Yang WE, Lee MS (2009) Comparison of clinical outcome in primary total hip arthroplasty by conventional anterolateral transgluteal or 2-incision approach. J Arthroplasty 24:528-532

27. Friedman LF, Furberg CD, DeMets DL (1998) Fundamentals of clinical trials, 3rd edn. Springer, New York, USA

28. Bland M (2006) An introduction to medical statistics, 3rd edn. Oxford University Press, Oxford

29. Yoon TR, Park KS, Song EK, Seon JK, Seo HY (2009) New twoincision minimally invasive total hip arthroplasty: comparison with the one-incision method. J Orthop Sci 14:155-160

30. Woolson ST, Mow CS, Syquia JF, Lannin JV, Schurman DJ (2004) Comparison of primary total hip replacements performed with a standard incision or a mini-incision. J Bone Joint Surg 86:1353-1358

31. Noble PC, Johnston JD, Alexander JA et al (2007) Making minimally invasive THR safe: conclusions from biomechanical simulation and analysis. Int Orthop 31(Suppl 1):S25-28

32. Speranza A, Iorio R, Ferretti M, D'Arrigo C, Ferretti A (2007) A lateral minimal-incision technique in total hip replacement: a prospective, randomised, controlled trial. Hip Int 17:4-8 
33. Procyk S (2007) Initial results with a mini-posterior approach for total hip arthroplasty. Int Orthop 31(Suppl 1):S17-S20

34. Sakamoto K, Arakawa H, Mita S et al (1994) Elevation of circulating interleukin 6 after surgery: factors influencing the serum level. Cytokine 6:181-186

35. Ogonda L, Wilson R, Archbold P et al (2005) A minimal-incision technique in total hip arthroplasty does not improve early postoperative outcomes. A prospective, randomized, controlled trial. J Bone Joint Surg 87:701-710

36. Laffosse JM, Chiron P, Molinier F, Bensafi H, Puget J (2007) Prospective and comparative study of the anterolateral miniinvasive approach versus minimally invasive posterior approach for primary total hip replacement. Early results. Int Orthop 31:597-603

37. Jerosch J, Theising C, Fadel ME (2006) Antero-lateral minimal invasive (ALMI) approach for total hip arthroplasty technique and early results. Arch Orthop Trauma Surg 126:164-173

38. Howell JR, Masri BA, Duncan CP (2004) Minimally invasive versus standard incision anterolateral hip replacement: a comparative study. Orthop Clin N Am 35:153-162

39. Archibeck MJ, White RE Jr (2004) Learning curve for the twoincision total hip replacement. Clin Orthop Relat Res 429:232-238

40. Swanson TV (2007) Posterior single-incision approach to minimally invasive total hip arthroplasty. Int Orthop 31(Suppl 1):S1-S5

41. Desser DR, Mitrick MF, Ulrich SD, Delanois RE, Mont MA (2010) Total hip arthroplasty: comparison of two-incision and standard techniques at an AOA-accredited community hospital. J Am Osteopath Assoc 110:12-15

42. Pagnano MW, Leone J, Lewallen DG, Hanssen AD (2005) Twoincision THA had modest outcomes and some substantial complications. Clin Orthop Relat Res 441:86-91

43. Bal BS, Haltorn D, Aleto T, Barrett M (2005) Early complications of primary total hip replacement performed with a two-incision minimally invasive technique. J Bone Joint Surg 87-A:2432-2438

44. Goosen JH, Kollen BJ, Castelein RM, Kuipers BM, Verheyen CC (2010) Minimally invasive versus classic procedures in total hip arthroplasty: a double-blind randomized controlled trial. Clin Orthop Relat Res. March 30 [Epub ahead of print]

45. Laffosse JM, Chiron P, Accadbled F, Molinier F, Tricoire JL, Puget J (2006) Learning curve for a modified Watson-Jones minimally invasive approach in primary total hip replacement: Analysis of complications and early results versus the standardincision posterior approach. Acta Orthop Belg 72:693-701

46. Berry DJ, Berger RA, Callaghan JJ et al (2005) Minimally invasive total hip arthroplasty. Development, early results and a critical analysis. J Bone Joint Surg 85-A:2235-2245
47. Wenz JF, Gurkan I, Jibodh SR (2002) Mini-incision total hip arthroplasty: a comparative assessment of perioperative outcomes. Orthopedics 25:1031-1043

48. Mow CS, Woolson ST, Ngarmukos SG, Park EH, Lorenz HP (2005) Comparison of scars from total hip replacements done with a standard or a mini-incision. Clin Orthop Relat Res 441:80-85

49. Yang C, Zhu Q, Han Y et al (2009) Minimally-invasive total hip arthroplasty will improve early postoperative outcomes: a prospective, randomized controlled trial. Ir J Med Sci 179(2):285289

50. Lawlor M, Humphreys P, Morrow E et al (2005) Comparison of early postoperative functional levels following total hip replacement using minimally invasive versus standard incisions. A prospective randomized blinded trial. Clin Rehabil 19:465-474

51. Kim YH (2006) Comparison of primary total hip arthroplasties performed with a minimally invasive technique or a standard technique. A prospective and randomized study. J Arthroplasty 21:1092-1098

52. Rittmeister M, Peters A (2006) Vergleich des hüftgelenkersatzes über eine posteriore miniinzision oder einen klassischen anterolateralen zugang. Orthopade 35:716-722

53. Leuchte S, Luchs A, Wohlrad D (2007) Measurement of ground reaction forces after total hip arthroplasty using different surgical approaches. Z Orthop 145:74-80

54. Kubeš J, Landor I, Podškubka A, Majernícek M, Vcelák J (2009) Total hip replacement from a MIS-AL approach (comparison with a standard anterolateral approach). Acta Chir Orthop Traumatol Cech 76:288-294

55. Szendrõi M, Sztrinkai G, Vass R, Kiss J (2006) The impact of minimally invasive total hip arthroplasty on the standard procedure. Int Orthop 30:167-171

56. Pospischill M, Kranzl A, Attwenger B, Knahr K (2010) Minimally invasive compared with traditional transgluteal approach for total hip arthroplasty: a comparative gait analysis. J Bone Joint Surg 92-A:328-337

57. Laffosse JM, Chiron P, Tricoire JL, Giordano G, Molinier F, Puget J (2007) Prospective and comparative study of minimally invasive posterior approach versus standard posterior approach in total hip replacement. Rev Chir Orthop Reparatrice Appar Mot 93:228-237

58. Wohlrab D, Hagel A, Hein W (2004) Advantages of minimal invasive total hip replacement in the early phase of rehabilitation. Z Orthop 142:685-690

59. Pflüger G, Junk-Jantsch S, Schöll V (2007) Minimally invasive total hip replacement via the anterolateral approach in the supine position. Int Orthop 31(Supp11):S7-S11 